\title{
Gold Price Prediction Method Based on Improved PSO-BP
}

\author{
Yan Wang ${ }^{1, a,{ }^{*}}$, Liguo Zhang ${ }^{2}$, Yongfu Liu ${ }^{2}$ and Jun Guo ${ }^{1}$ \\ ${ }^{1}$ Dept. of computer, North China Electric Power University, Baoding, Hebei, \\ 071003, China \\ ${ }^{2}$ College of Information Science \& Technology, Agricultural University of Hebei \\ Hebei Baoding, 071001, China \\ ${ }^{a}$ Wangyan1206@126.com
}

\begin{abstract}
Aimed at the highly nonlinear and uncertainty of gold price changes, a new method for gold price predition based on improved PSO-BP is proposed. By introducing mutation operation and adaptive adjust of inertia weight, the problem of easy to fall into local optimum, premature, low precision and low later interation efficiency of PSO are solved. By using the improved PSO to optimiaze BP neural network's parameters, the learning rate and optimization capability of conventional BP are effectively improved. The simulation results of gold price prediction show that the predict accuracy of the new method is significantly higher than that of conventional BP neural network and wavelet neural network method. And the method is effective and feasible.
\end{abstract}

Keywords: Particle swarm optimization (PSO), mutation, adaptive adjust, Backpropagation neural network, Gold price prediction

\section{Introduction}

For a long time, gold has caught a global attention for its functions as a measure of value, means of circulation, payment instruments, reserve assets and the world's monetary. It has become a major project of the theoretical and empirical research that how to effectively predict the price of gold. Many scholars, at home and abroad, have made much related research and constructed a number of very valuable theoretical hypothesis and prediction models, such as ARMA model, ARIMA model, adaptive filtering prediction models, varying coefficient regression model, BP neural network model and so on. Ref [1] has applied wavelet neural network to predict the gold price. Zeng lian and Ma Diandi established the gold price forcast simulation model based on BP neural network optimized by projection pursuit. Wang Yan used cross validation and the coefficient regression model and multiple linear regression model to focast gold pice. The main affect factors are U.S. Dollar index, Oil prices, silver prices, DOW index, OECD leading index, European stock markets, etc. However, the gold price fluctuation trend shows a high nonlinearity and uncertainty, which leads to accurately predict the gold price is difficult. Artificial neural network prediction method can better handle the nonlinear and uncertain problems, but it also has many shortcomings, such as: model training slow; time and space complexity is high; easy to fall into local optimum.

Particle swarm optimization (PSO) is a population based stochastic optimization technique developed by Dr. Eberhart and Dr. Kennedy in 1995. As a group intelligent search algorithm, it through population cooperation and competition between the particles to guide group search. And it has many merits, such as parallel global search, the model is simple and convenient, few parameters need to be

${ }^{*}$ Corresponding Author 
adjusted, convergence is fast and easy implementation. Thus, using PSO algorithm for BP Neural network pre-search can overcome the deficiencies of BP algorithm. However, when there are more locally optimums, standard PSO algorithm also easy to fall into local optimum. Many researchers have made studies for improving the PSO algorithm and achieved some success. The paper proposed the grain yield prediction method based on improved PSO-BP, and the prediction results show that the prediction model can effectively improve the prediction accuracy.

\section{BP Neural Network}

Artificial neural networks are powerful tool for prediction of nonlinearities. These mathematical models comprise individual processing units called neurons that resemble neural activity. Each processing units sums weighted inputs and then applies a linear or nonlinear function to the resulting sum to determine the output. The neurons are arranged in layers and are combined through excessive connectivity. With hierarchical feed forward network architecture, the backpropagation network has received most attention.

Typically, three-layer BP neural network (input layer, hidden layer and output layer) can realize the function mappings of $n$ independent variables and the $m$ dependent variables. In the study of BP neural network, the main features are forward transformer of input signal and back-propagation of error. Network's weights and threshold values are adjusted according to the prediction error.

The signal inputted from outside spreads to the output layer and gives the result through processing layer for layer of neurons in input layer and hidden layer. If the expected output can't be obtained in output layer, it shifts to the conversed spreading processing and the true value and the error outputted by network will return along the coupled access formerly. The error is reduced by modifying contacted weight value of neurons in every layer and then it shifts to the positive spreading processing and revolves iteration until the error is smaller the given value. The topological of BP neural network is shown in Figure 1.

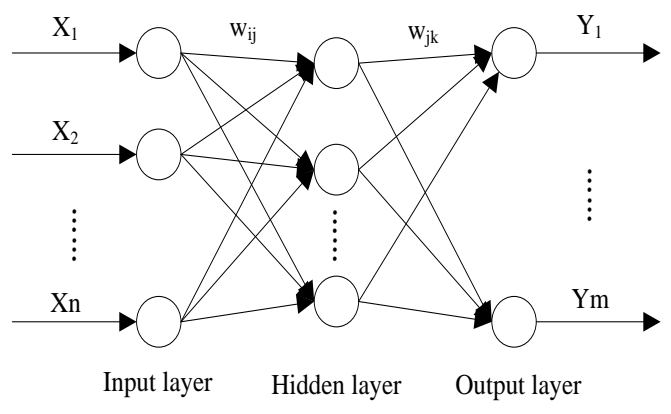

Figure 1. The Topological of BP Neural Network

Here, $X_{1}, X_{2}, \cdots, X_{n}$ are the input values of neural network, $Y_{1}, Y_{2}, \cdots, Y_{n}$ are the predictive values, $\omega_{i j}$ and $\omega_{j k}$ are network's weights. Before using, the first task is to train the network. The training process included the following steps.

Step 1: Initialize the network. According to the input and output of actual system, determine the numbers of input layer nodes, hidden layer nodes and output layer nodes, initialize $\omega_{i j}, \omega_{j k}$ and the threshold value of both hidden layer and output layer, and set the learning rate and the neuron activation function. 
Step 2: Calculate the hidden layer output based on formula (1).

$$
H_{j}=f\left(\sum_{i=1}^{n} \omega_{i j} x_{i}-a_{j}\right) j=1,2, \cdots, l
$$

Where, ${ }^{l}$ is the number of nodes in hidden layer, $a_{j}$ is the threshold value and $f$ is the activation function of hidden layer. In this paper, we select formula (2) as $f$.

$$
f(x)=\frac{1}{1+e^{-x}}
$$

Step 3: Calculate the output value of output layer based on formula (3).

$$
O_{k}=\sum_{j=1}^{l} H_{j} \omega_{j k}-b_{k} k=1,2, \cdots, m
$$

Where, $b_{k}$ is the threshold value of output layer node.

Step 4: Calculate the prediction error according to the network predicted output and the desired output.

$$
e_{k}=Y_{k}-O_{k} k=1,2, \cdots, m
$$

Step 5: Update the connection weights by the prediction error $e_{k}$.

$$
\begin{gathered}
\omega_{i j}=\omega_{i j}+\eta H_{j}\left(1-H_{j}\right) x(i) \sum_{k=1}^{m} \omega_{j k} e_{k} i=1,2, \cdots, n ; j=1,2, \cdots, l \\
\omega_{j k}=w_{j k}+\eta H_{j} e_{k} \quad j=1,2, \cdots, l ; k=1,2, \cdots, m
\end{gathered}
$$

Here, $\eta$ is the learning rate.

Step 6: Update the threshold value based on formula (7) and (8).

$$
\begin{gathered}
a_{j}=a_{j}+\eta H_{j}\left(1-H_{j}\right) \sum_{k=1}^{m} \omega_{j k} e_{k} j=1,2, \cdots, l \\
b_{k}=b_{k}+e_{k} k=1,2, \cdots, m
\end{gathered}
$$

Step 7: Determine whether the iterative ends, and if not, return to Step 2.

\section{PSO Algorithm and its Improvement}

\subsection{Standard PSO Algorithm}

Given in a Q-dimensional search space, there is a particle community composed of $n$ particles. And the relevant parameters of $i$-th particle are denoted as follows: the position vector is denoted by $x_{i}=\left(x_{i 1}, x_{i 2}, \cdots, x_{i Q}\right), i=1,2, \cdots, n$. The flying speed is denoted by $v_{i}=\left(v_{i 1}, v_{i 2}, \cdots, v_{i Q}\right)$. Up to now, the searched optimal location of $i$-th particle is denoted by $p_{i}=\left(p_{i 1}, p_{i 2}, \cdots, p_{i Q}\right)$ (Namely $\left.\mathrm{P}_{\text {best }}\right)$. the searched optimal location of the whole particles community is denoted by $p_{g}=\left(p_{g_{1}}, p_{g_{2}}, \cdots, p_{g Q}\right)$ (Namely $\left.\mathrm{G}_{\text {best }}\right)$. To search the optimal solution in Q- 
dimensional space is to search the particle in best position. According the three principles, maintain its inertia, maintain its optimal position and maintain community optimal position, the particle updates its status during the moment.

In every iteration, the particles update their velocity and position by formula(9).

$$
\begin{aligned}
& v_{i d}^{k+1}=\omega v_{i d}^{k}+c_{1} \xi\left(P_{b e t}^{k}-x_{i d}^{k}\right)+c_{2} \eta\left(G_{b e s t}^{k}-x_{i d}^{k}\right) \\
& \left\{\begin{array}{l}
v_{i d}^{k+1}=v_{\text {max }} \quad, \quad v_{i d}^{k+1}>v_{\text {max }} \\
v_{i d}^{k+1}=-v_{\text {max }}, v_{i d}^{k+1}<-v_{\text {max }}
\end{array}\right. \\
& x_{i d}^{k+1}=x_{i d}^{k}+\lambda v_{i d}^{k+1}
\end{aligned}
$$

Here, $\omega$ denotes inertia weight and used to maintain the original rate coefficients. $c_{1}$ and $c_{2}$ denote learning factor and acceleration coefficients, respectively. $\xi$ and $\eta$ are the uniformly distributed random numbers during 0 and 1. $\lambda$ is constraint factor. $\left[-\mathrm{v}_{\max }, \mathrm{v}_{\max }\right]$ is velocity range for each dimension of particle. Standard PSO algorithm flow is shown in Figure 2.

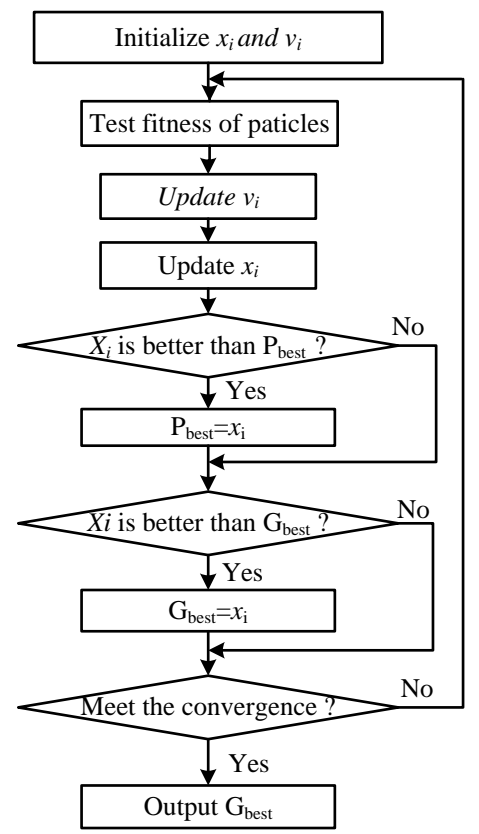

Figure 2. Standard PSO Algorithm Flow

\subsection{The Improvement of PSO}

For the standard PSO algorithm is easy to fall into local optimum problem, the paper introduced the mutation operation to PSO algorithm. The basic idea is to reinitialize the particle after each update with a certain probability. The adaptive mutation operation method for $\mathrm{i}$-th particle is as follows:

$$
x_{i j}=\left\{\begin{array}{cc}
x_{\mathrm{ij}} & r<=\mathrm{P} \\
x_{\text {random }} & r>\mathrm{P}
\end{array}\right.
$$

Here, ${ }^{x_{i j}}$ denotes the $\mathrm{j}$-th component of particle ${ }^{x_{i}}, \mathrm{P}$ denotes mutation probability, $r$ the uniformly distributed random numbers during 0 and $1,{ }^{x_{\text {random }}}$ 
denotes random number during individual maximum and minimum position of particle.

Research shows that the liner decreasing inertia weigh can better balance the global search ability and local search ability. The paper adopts the following method to get inertia weight value.

$$
\omega(k)=\omega_{\text {sat }}+\left(\omega_{\text {satat }}-\omega_{\text {end }}\right) *\left(2 * k / T_{\text {max }}-\left(k / T_{\text {max }}\right)^{2}\right)
$$

Here, $\omega_{\text {start }}$ denotes initial inertia weight $; \omega_{\text {end }}$ denotes inertia weight of maximum iteration number, $k$ denotes current iteration number, $T_{\max }$ denotes maximum iteration number.

\subsection{The Improved PSO-BP Network}

BP neural network learning process is the update process of the connection weights and thresholds of the network. The purpose of using PSO algorithm optimize BP neural network is to get better network initial weights and thresholds. The basic idea is to use the position of each individual particle in PSO to represent all of the initial network connection weights and threshold parameters. Then take the individual initialized BP neural network prediction error as the individual's fitness value and through the particle network optimization to find the best initial weights and thresholds.

The detailed algorithm can be summarized as follows:

1)Design and initialize the network, normalize the samples.

2)Initialize PSO, such as, population size, particle structure, location and speed.

3)Calculate fitness value of each particle. The paper takes formula(4) as particle fitness function.

$$
f \text { itness }=\sum_{i=1}^{N}\left|y_{i}-d_{i}\right|
$$

Here: $N$ denotes training sample number, $d_{i}$ denotes the desired output of $i$-th sample. $y_{i}$ denotes network computing values of $i$-th sample.

4)According to the fitness value of each particle, update its personal best position $P_{\text {best }}$ and global best position $G_{\text {best }}$.

5) According to formula (9), adjust the position and velocity of particle.

6) According to formula (10), make adaptive mutation operation.

7) If the convergence criteria is met (the number of iteration is reached or the error can accepted), stop iteration. And the $G_{\text {best }}$ is the initial parameter values of BP network, Through further learning and training of BP algorithm can form the predict model. Otherwise, go to step 3 for the next iteration.

\section{Gold Price Prediction based on Improved}

According to previous studies and related references, there are many factors affecting Gold price including Dow Jones index, America consumer price index,USA federal funds rate, American world gold reserves and oil prices, OECD leading index and other factors. In the proposed prediction model, take Dow Jones 
index, America consumer price index,America dollar nominal effective exchage rate, world gold reserves, USA federal funds rate and oil prices as inputs and take Gold price as the output. Thus the BP neural network structure is shown in Figure 3. Then, take the collected sample data from 1973 to 2000 as training sample data and the sample data from 2001 to 2006 as testing sample data. In the test, the relevant parameters of PSO algorithm are as follows: the number of iteration is 50, population size is $20, \mathrm{c} 1=1.49445, \mathrm{c} 2=1.49445$ and the length of each particle is 41 . Each generation best individual fitness curve of improved PSO algorithm optimization process is shown in Figure 4. The training error curves of the the BP network optimized by improved PSO are shown in Figure 5. The contrast curve of golde price of forecast results and the real value form 2001 to 2006 years is shown in Figure 6. The obtained optimal initial weights and thresholds of BP neural network is shown in Table 1. Predictions contrast of the proposed method and other method for predicted gold price from 2001 to 2006 is shown in Table 2.

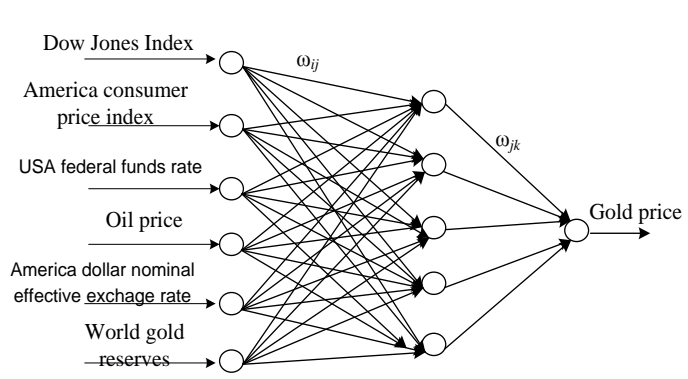

Figure 3. BP Network Structure for Gold Price Prediction

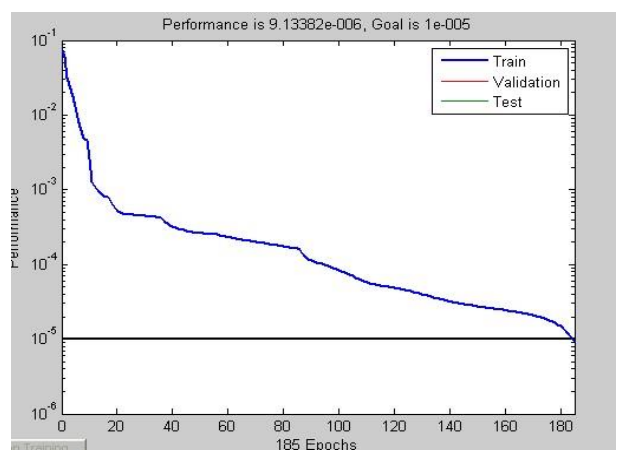

Figure 5. Train Error of Optimized BP

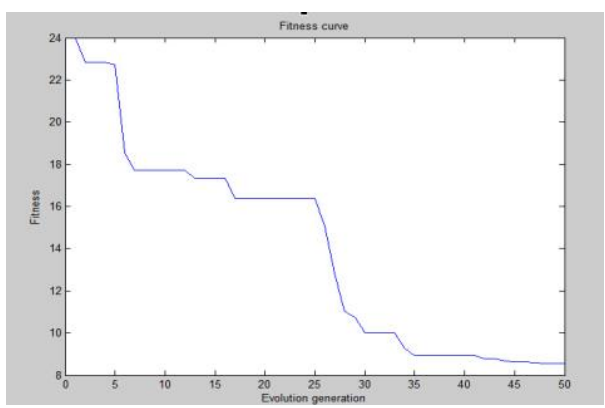

Figure 4. Best Individual Fitness Curve of Improved PSO

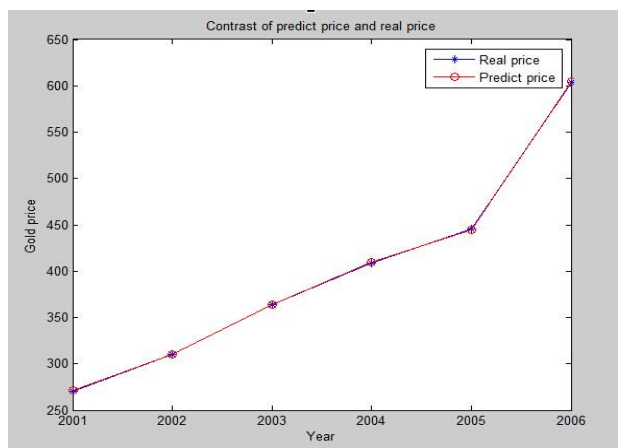

Figure 6. Cure of Predicted Price and Real Price

Table 1. Optimal Initial Weights and Thresholds

\begin{tabular}{|c|c|c|c|c|c|c|c|}
\hline $\begin{array}{c}\text { The Initial weights } \\
\text { between input layer nodes } \\
\text { and hidden layer nodes }\end{array}$ & -2.6303 & 0.3696 & -3.6920 & -1.5805 & -3.3660 & $\ldots$ & -2.8345 \\
\hline $\begin{array}{c}\text { The thresholds of hidden } \\
\text { layer nodes }\end{array}$ & 0.9963 & 1.5325 & -5.0000 & -0.9072 & 1.6255 & - & - \\
\hline $\begin{array}{c}\text { The Initial weights } \\
\text { between output layer } \\
\text { nodes and hidden layer } \\
\text { nodes }\end{array}$ & -0.0261 & 0.4151 & -1.7188 & 0.6047 & -0.1781 & - & - \\
\hline $\begin{array}{c}\text { The thresholds of output } \\
\text { layer nodes }\end{array}$ & -1.6850 & - & - & - & - & - & - \\
\hline
\end{tabular}


Table 2. Prediction Contrast

\begin{tabular}{cccccccc}
\hline \multirow{2}{*}{ Year } & $\begin{array}{c}\text { Real } \\
\text { Gold } \\
\text { Price }\end{array}$ & \multicolumn{2}{c}{ BP network model } & \multicolumn{2}{c}{ Ref } & \multicolumn{2}{c}{$\begin{array}{c}\text { Improved PSO-BP } \\
\text { network model }\end{array}$} \\
\cline { 3 - 8 } & & Price & $\begin{array}{c}\text { Relative } \\
\text { error/(\%) }\end{array}$ & $\begin{array}{c}\text { Predicted } \\
\text { price }\end{array}$ & $\begin{array}{c}\text { Relative } \\
\text { error/(\%) }\end{array}$ & $\begin{array}{c}\text { Predicted } \\
\text { price }\end{array}$ & $\begin{array}{c}\text { Relative } \\
\text { error/(\%) }\end{array}$ \\
\hline 2001 & 270.95 & 282.9927 & 4.4 & 264.1546 & 2.5 & 272.5977 & 0.6 \\
2002 & 309.98 & 299.7074 & 3.3 & 311.1185 & 0.4 & 309.4458 & 0.2 \\
2003 & 363.44 & 374.7952 & 3.1 & 357.5867 & 1.6 & 362.9662 & 0.1 \\
2004 & 409.23 & 420.9413 & 2.9 & 404.5547 & 1.1 & 409.0363 & 0.1 \\
2005 & 444.88 & 433.0574 & 2.7 & 442.7708 & 0.5 & 445.0409 & 0.1 \\
2006 & 604.27 & 610.0051 & 0.9 & 605.3447 & 0.2 & 603.7613 & 0.1 \\
\hline
\end{tabular}

As can be seen from Figure 4, the best individual fitness obtained by improved PSO-BP neural network method has better optimization capability in evolution than that of standard BP neural network. Under the same training accuracy and by comparing figure 5 , it can be seen that the proposed method can meet the convergence $(0.00001)$ at $185^{\text {th }}$ generation and obviously superior to conventional BP network $\left(30588^{\text {th }}\right.$ generation) and $\operatorname{Ref}\left[\operatorname{method}\left(3690^{\text {th }}\right.\right.$ generation $)$. By data comparison of Table 2,the prediction accuracy of improved PSO-BP method is superior to that of conventional BP network and Ref method for the same statistics data. The maximum relative error of BP network method, Ref[method and improved PSO-BP method are 4.4\%, 2.5\% and 0.6, respectively. The Mean absolute error of the proposed method and Ref method is 0.5865 and 3.6077.From the comparison, it can be seen that the proposed gold price prediction method is effective and feasible.

\section{Conclusion}

Gold futures price is the combined result of a large number of factors. Because of high nonlinear, high noise and because the factors is determine difficultly, the prediction is complex and difficult. Traditional methods of predicting the price of gold have emphasized the intrinsic value of gold, or dependent on the linear relationship between the prices of gold. The limitations are obvious, which leads to the low prediction precision. The paper proposed the improved PSO-BP based gold price prediction method, which optimized the BP neural network parameters through improved PSO and effectively improved the overall learning ability and overcome the problem of easy to fall into local optimum. The test results for 2001-2006 gold price shows the proposed method is significantly better than BP neural network method and wavelet neural network based method, and has good application prospects for gold price forecast.

\section{Acknowledgements}

This work was supported by the Fundamental Research Funds for the Central Universities(2014MS132), Hebei Science Research and Development Project of China (13215206) and Technology Foundation of Agricultural University of Hebei (LG20130804).

\section{References}

[1] K. Y. Zhang, Yu and T. Li, "Application of wavelet neural network in prediction of gold price", Comput. Eng. Applic, vol. 46, pp. 224-226, (2010).

[2] L. Zeng, D. D. Ma and Z. X. Liu, "Gold price forecast based on improved BP neural network", Comput. Simul., vol. 27, (2010), pp. 200-203. 
[3] Q. Zhang, J. H. Ma and Y. Wang, "Study on forecasting of gold price based on varying-coefficient regression model”, Key Engineering Materials, vol. 456, (2011), pp. 1398-1403.

[4] L. Chen, "Gold price forecasting model based on projection pursuit and neural network", Comput. Simul, vol. 30, (2013), pp. 354-357.

[5] F. Wang, T. Ma and X. Ma, "Gold price forecasting model based on regression with support vector machine for particle swarm optimization", J. Lanzhou Univ. Technol., vol. 39, (2013), pp. 65-69.

[6] L. P. Xu and M. Z. Luo, "Short-term analysis and prediction of gold price based on ARIMA model", Finance Econ., vol. 1, (2011), pp. 26-34.

[7] X. Wei, "Sensor temperature compensation technique simulation based on BP neural network", Telkomnika, vol. 11, (2013), pp. 3304-3313.

[8] B. H. M. Sadeghi,, “A BP-neural network predictor model for plastic injection molding process”, J. Mater. Process. Technol., vol.103, (2000), pp. 411-416.

[9] J. Kennedy and R. Eberhart, "Particle swarm optimization. Proceedings of the International Conference on Neural Networks", vol. 4, November 27-December 1, 1995, Perth, WA., USA., pp: 1942 -1948, (1995).

[10] J. H and H. Guo, "A modified particle swarm optimization algorithm”, Telkomnika, vol. 11, (2013), pp. 6209-6215.

[11] X. Q. Yan, Wu and H. Liu, "Orthogonal particle swarm optimization algorithm and its application in circuit design”, Telkomnika, vol. 11, (2013), pp. 2926-2932.

[12] C. Y. Zhao, Z. B. Yan and X. G. Liu, "Improved adaptive parameter particle swarm optimization algorithm”, J. Zhejiang Univ. (Eng. Sci.), vol. 39, (2011), pp. 1039-1042.

\section{Author}

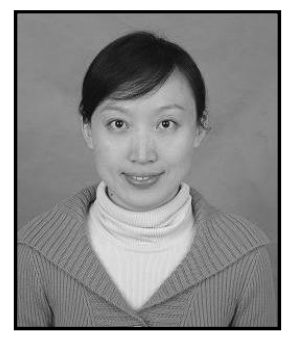

Yan Wang, she was born on 12/6/1981. and obtained the B.Eng. degree and the M.Eng. degree in computer software and theory specialty from School of Control and Computer Engineering at North China Electric Power University china at 2004 and 2007 respectively. Now, she works as lecturer at School of Control and Computer Engineering at North China Electric Power University china. his research interests concentrate on the development of software, Computer application and Artificial intelligence, etc. 